\title{
Human Parvovirus B19: ubiquitous but not necessarily benign
}

Jun Yi Sim a,b, Luan-Yin Changa, Jong-Min Chenª, Ping-Ing Leea, Li-Min Huang ${ }^{a}$, Chun-Yi Lua

aDivision of Pediatric Infectious Diseases, Department of Pediatrics, National Taiwan University Hospital, Taipei, Taiwan bDepartment of Pediatrics, Taipei Tzu Chi Hospital, Buddhist Tzu Chi Medical Foundation

\section{More than erythema infectiosum}

- Human parvovirus B19, a member of the Parvoviridae family, has been known to cause self-limiting diseases including erythema infectiosum (fifth disease) and arthropathy in healthy subjects.

- However, there is limited understanding on symptoms, physical findings, and clinical course, especially in patients with comorbidities.

- We retrospectively analyzed patients who tested positive for parvovirus B19 by real-time PCR in blood in our institution.

- We aim to identify physical findings that were correlated with severe disease.

\section{Methods}

- From January 2009 to December 2017, patients who were positive for parvovirus B19 in blood via real-time PCR were enrolled.

\section{- Complicated patients were} defined as having received a blood product transfusion.

Young, N.S., Brown, K.E. Parvovirus B19. N Engl J Med, 2004,350:586-97

2. Wolfromm, A., et. al., Spectrum of adult Parvovirus B19 infection according to the underlying predisposing condition and proposals for clinical practice. Br J Haematol, 2015, 170(2) 192-9

\section{Results}

- 26 patients (15 male/11 female) had positive parvovirus B19 PCR in blood.

- Median age was 8.8 years.

- Prior to a positive PCR test, patients' initial diagnosis were as follows:

\section{Initial diagnosis}

Erythema infectiosum

Fever of unknown origin (FUO)

Acute tonsillitis

Atypical infection

Scarlet fever

Hydrops fetalis

Viral myocarditis

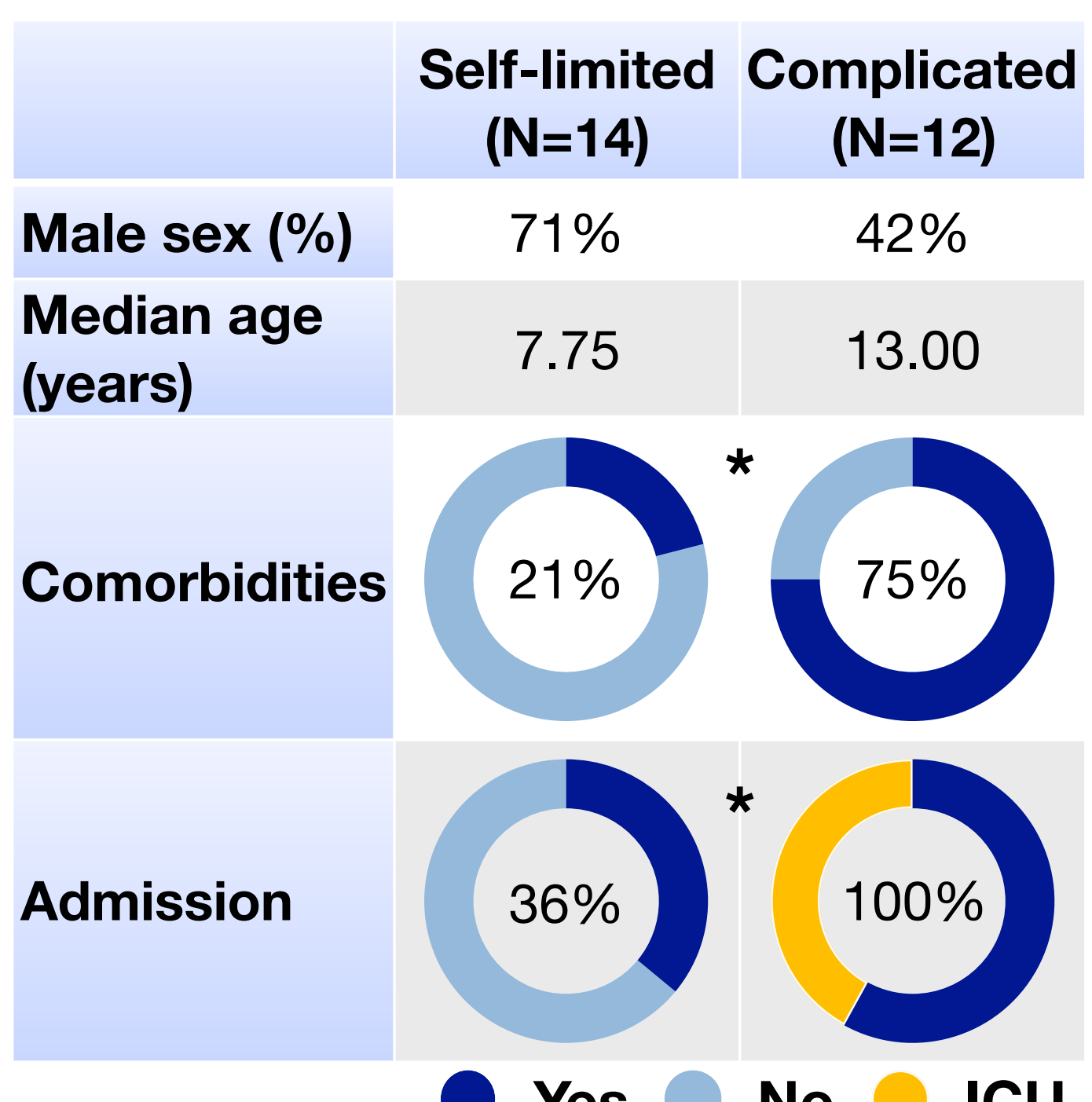

$\mathbf{N}$

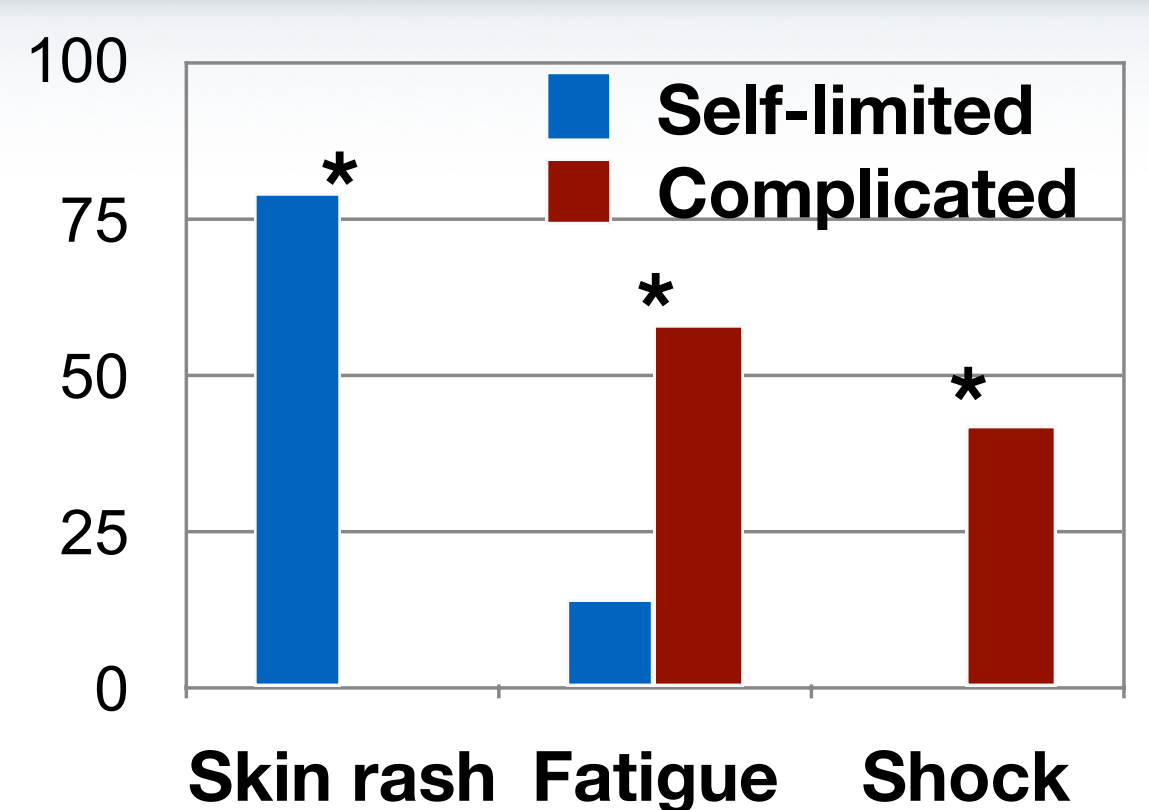

6

100

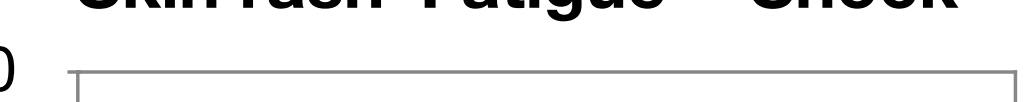

75

6

3

2

1

1

$$
0
$$

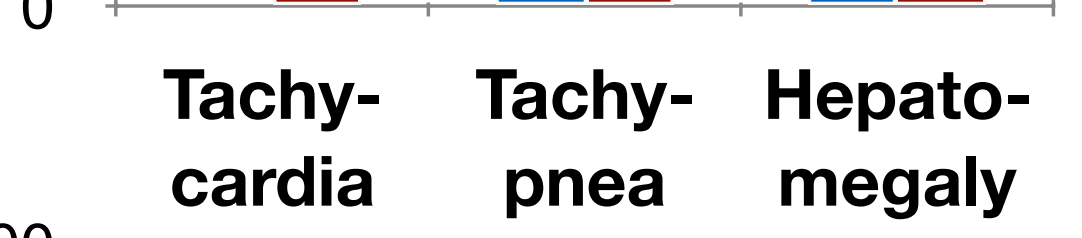

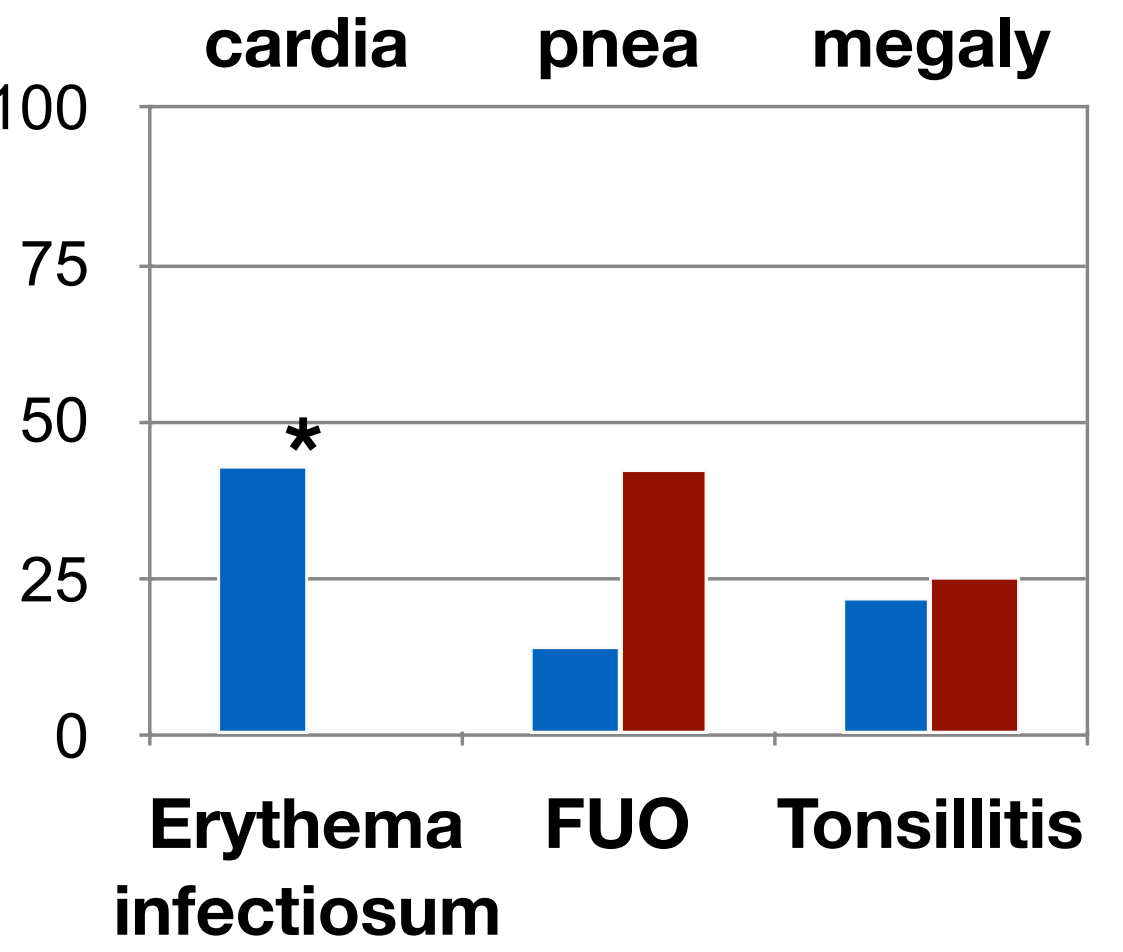
infectiosum

Table 1 (left) and Figure 1 (above): Characteristics and comparison of symptoms, physical findings, and initial diagnosis between patients with selflimited and complicated disease

*Indicates statistically significant, $p<0.05$

Discussion

- This 8-year retrospective case series documents the wide spectrum of parvovirus B19 presentation in children and adults.

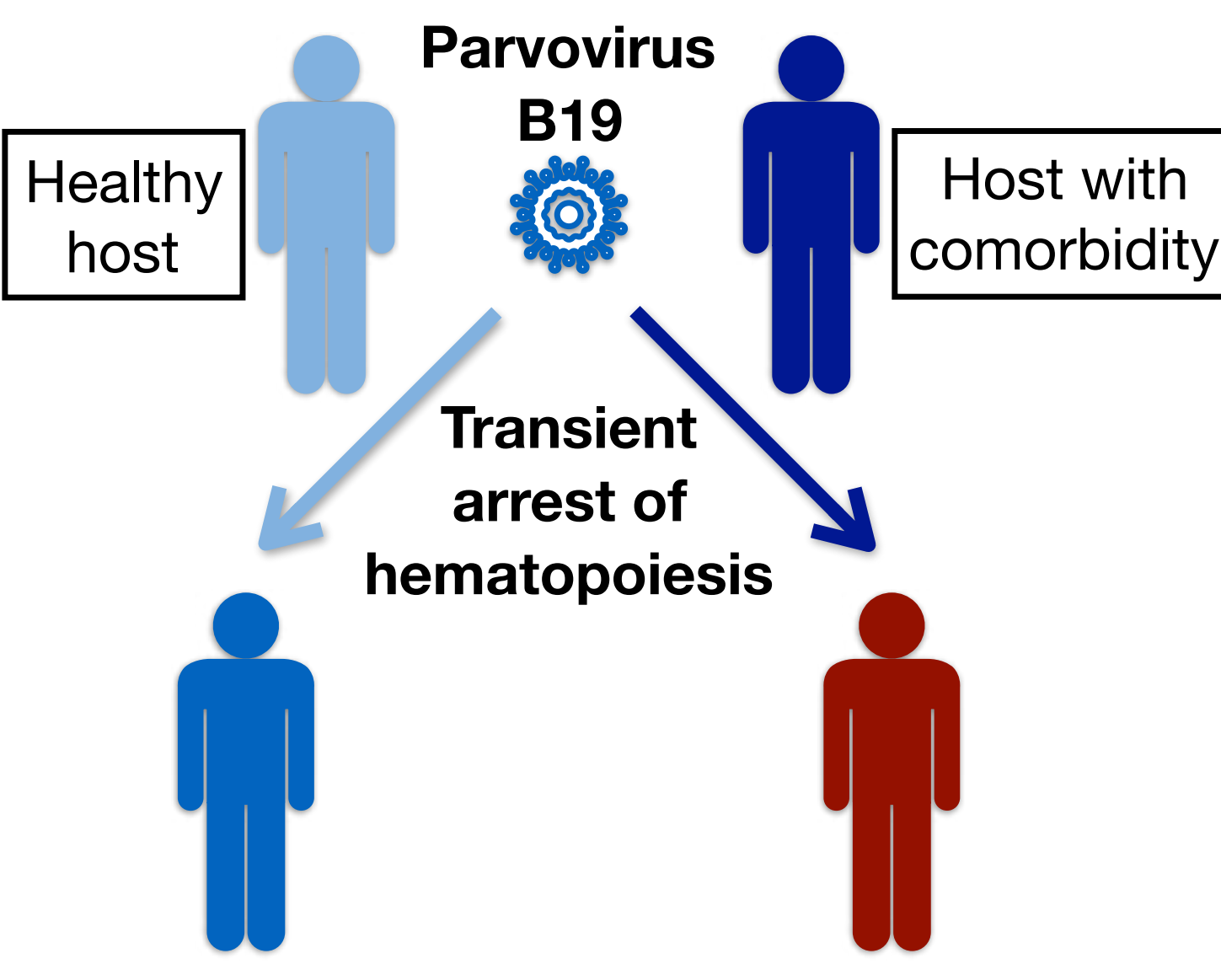

Self-limited - Transient reticulocytopenia but no clinical anemia

- Rash likely due to immune complex, viremia resolution

\begin{tabular}{|l|}
\hline \multicolumn{1}{|c|}{ Complicated } \\
- Aplastic crisis due to \\
short red cell lifespan \\
- Prolonged viremia \\
from immuno- \\
suppression \\
- Heart failure, hydrops \\
\hline
\end{tabular}

\section{Conclusions}

- Depending on host factors, the manifestations of parvovirus B19 infection can be multifaceted.

- Patients who required transfusion had physical findings that were significant compared to those with a self-limited course. 